\title{
CONTROL OF LINEAR SYSTEMS USING DYNAMIC OUTPUT CONTROLLERS
}

\author{
Anna Filasova and Dusan Krokavec \\ Department of Cybernetics and Artificial Intelligence, Technical University of \\ Košice, Faculty of Electrical Engineering and Informatics, \\ Letna 9, 04200 Kosice, Slovakia
}

\begin{abstract}
The paper deals with the problem of control of continuous-time linear systems by the dynamic output controllers of order equal to the plant model order. The design procedure is based on a solution of the set of linear matrix inequalities and ensures the closed-loop stability using Lyapunov approach. Numerical examples are given to illustrate the design procedure and relevance of the methods as well as to validate the performances of the proposed approach
\end{abstract}

\section{KEYWORDS}

Linear systems, dynamic output controllers, Lyapunov function, linear matrix inequality

\section{INTRODUCTION}

In practice, online measurements of all state variables of a process are rarely available and since only their observable outputs are accessible, the output feedback control laws have to be considered. Since, really, the system dynamic may be affected by unmeasurable disturbances the $\mathrm{H}_{\infty}$ approach is proposed to be used in the static and dynamic output feedback control law design.

The static output feedback problem seems to be one of the most important questions in linear control system design, see, e.g. [1], [2], [3], [4] and the reference therein. Because of the importance of these kind control systems, considerable attention was dedicated to the study of suitable design methods. Reflecting the fact that the static output feedback stabilization is a concave-convex problem [5], the design conditions based on solution of various mutually coupled matrix equations or coupled linear matrix inequalities (LMI) was discussed, e.g., in [6], [7].

Exploiting the approaches which potentially allow converting dynamic output controller synthesis into an LMI optimization problem, LMI computational technique has brought a tool to solve also this task. An iterative algorithm for designing the linear time-invariant dynamic output controllers of the prescribed structure was presented in [8], formulating the solution as an optimization based on LMIs in which either the Lyapunov matrix or the controller parameter matrix are alternately regarded as the optimization variables. Another iterative approach was proposed in [9], where a convexifying function is reduced in each iteration step to zero to guaranty the feasibility of the problem. Applying the controller parameter transformation and a mix of performance measures, the no recursive approach noted as the multi-objective synthesis of linear dynamic outputfeedback controllers is presented in [10], [11] where each objective is formulated relative to a

David C. Wyld et al. (Eds) : SAI, CDKP, ICAITA, NeCoM, SEAS, CMCA, ASUC, Signal - 2014 
variety of the closed-loop transfer function and more relaxed sufficient conditions are derived in terms of LMIs.

The aim of this paper is to compare the existing results in design of non-proper and proper dynamic output controllers, but above all to formulate a new design conditions based on the set LMIs and, as yet, one linear matrix equality (LME) the non-proper dynamic output control as well as to extend the methodology for the proper dynamic output control [12]. Applying to the multi-input and multi-output linear systems convexifying assumptions are solved by modifying the $\mathrm{H}_{2}$ control problem. The stability of the closed-loop system is ensured by finding a suitable Lyapunov matrix within a resolution of the proposed LMIs and LME structure.

The paper is organized in six sections. Following the introduction in Sec. 1, the considered structures of the dynamic output controllers are presented in Sec. 2. The main results are outlined in Sec. 3 and 4, formulating stability analysis and suitable design methods for the given types of output control by use of LMIs. In Sec. 5 the numerical example is given in order to discuss the performances and limitations of the proposed design methods and the last section draws some concluding remarks.

Throughout the paper, the notations are narrowly standard in such a way that $x^{T}, X^{T}$ denotes the transpose of the vector $x$ and matrix $X$, respectively, $X=X^{T}>0$ means that $X$ is a symmetric positive definite matrix, $\operatorname{rank}(\cdot)$ remits the rank of a matrix, $\operatorname{diag}[\cdot]$ designates a block diagonal matrix, the symbol $I_{n}$ indicates the $n$-th order unit matrix, $R$ denotes the set of real numbers and $R^{n}, R^{n \times r}$ refer to the set of all $n$-dimensional real vectors and $n \times r$ real matrices, respectively.

\section{PROBLEM FORMULATION}

The systems under consideration are continuous-time linear MIMO systems, described in the state-space form by the set of equations

$$
\begin{gathered}
\dot{\boldsymbol{q}}(t)=\boldsymbol{A} \boldsymbol{q}(t)+\boldsymbol{B} \boldsymbol{u}(t) \\
\boldsymbol{y}(t)=\boldsymbol{C} \boldsymbol{q}(t)
\end{gathered}
$$

where $q(t) \in R^{n}, u(t) \in R^{r}$ and $y(t) \in R^{m}$ are vectors of the system, input and output variables, respectively and the matrices $A \in R^{n \times n}, B \in R^{n \times r}, C \in R^{m \times n}$ are real matrices, provided that $(A, B)$ is stabilisable and $(A, C)$ is detectable.

It is assumed that the system is stabilized by the full order time-invariant be-proper dynamic output controller

$$
\begin{gathered}
\dot{\boldsymbol{p}}(t)=\boldsymbol{J} \boldsymbol{p}(t)+\boldsymbol{L} \boldsymbol{y}(t) \\
\boldsymbol{u}(t)=\boldsymbol{M p}(t)+\boldsymbol{N} \boldsymbol{y}(t)
\end{gathered}
$$

and by the full order time-invariant strictly proper dynamic output controller

$$
\begin{gathered}
\dot{p}(t)=\boldsymbol{J} \boldsymbol{p}(t)+\boldsymbol{L} \boldsymbol{y}(t) \\
\boldsymbol{u}(t)=\boldsymbol{M p}(t)
\end{gathered}
$$


where $p(t) \in R^{n}$ is the vector of the controller state variables, the controller matrices

$$
K^{o}=\left[\begin{array}{cc}
J & L \\
M & N
\end{array}\right], \quad K^{\cdot}=\left[\begin{array}{cc}
J & L \\
M & 0
\end{array}\right]
$$

$K^{o} \in R^{(n+r) \times(n+m)}, K^{o} \in R^{(n+r) \times(n+m)}$ has the prescribed structure with respect to the real matrices $J \in R^{n \times n}, L \in R^{n \times m}, M \in R^{r \times n}$ and $N \in R^{r \times m}$ or $0 \in R^{r \times m}$.

Considering that the plant (1), (2) is square, i.e., $r=m$, the objective is to present design conditions to expose the above described matrix parameters of the dynamic controllers.

\section{BI-PROPER DYNAMIC OUTPUT CONTROLLER}

To analyse the stability of the closed-loop system structure with the bi-proper dynamic output controller, the following form of the closed-loop system description can be introduced

$$
\begin{gathered}
{\left[\begin{array}{c}
\dot{q}(t) \\
\dot{p}(t)
\end{array}\right]=\left[\begin{array}{cc}
A+B N C & B M \\
L C & J
\end{array}\right]\left[\begin{array}{l}
q(t) \\
p(t)
\end{array}\right]} \\
y(t)=\left[\begin{array}{ll}
0 & I_{m}
\end{array}\right]\left[\begin{array}{cc}
0 & I_{m} \\
C & 0
\end{array}\right]\left[\begin{array}{c}
q(t) \\
p(t)
\end{array}\right]
\end{gathered}
$$

After introducing the notations

$$
\begin{gathered}
q^{o T}(t)=\left[\begin{array}{ll}
q^{T}(t) & p^{T}(t)
\end{array}\right] \\
A^{o}=\left[\begin{array}{ll}
A & 0 \\
0 & 0
\end{array}\right], \quad B^{o}=\left[\begin{array}{cc}
0 & B \\
I_{n} & 0
\end{array}\right], \quad C^{o}=\left[\begin{array}{cc}
0 & I_{n} \\
C & 0
\end{array}\right], \quad I^{o}=\left[\begin{array}{ll}
0 & I_{m}
\end{array}\right]
\end{gathered}
$$

where, in general, $A^{o} \in R^{2 n \times 2 n}, B^{o} \in R^{2 n \times(n+r)}, C^{o} \in R^{(n+m) \times 2 n}, I^{o} \in R^{m \times(n+m)}$, the closed-loop state-space equations takes the form

$$
\begin{array}{r}
\dot{q}^{\circ}(t)=\left(A^{\circ}+B^{\circ} K^{\circ} C^{\circ}\right) q^{\circ}(t) \\
y^{o}(t)=I^{o} C^{o} q^{o}(t)
\end{array}
$$

In the sequel, so it is supposed that $\left(A^{o}, B^{o}\right)$ is stabilizable, $\left(A^{o}, C^{o}\right)$ is detectable [15] and the matrix product $C^{\circ} B^{\circ}$ is nonzero matrix.

\section{Theorem 1}

The closed-loop system consisting of the plant (1), (2) with the be-proper dynamic output controller (3)-(4) is stable if there exist a symmetric positive definite matrix $Q^{o} \in R^{2 n \times 2 n}$, a regular matrix $H^{o} \in R^{(n+m) \times(n+m)}$ and a matrix $Y^{o} \in R^{(n+r) \times(n+m)}$ such that

$$
\begin{gathered}
Q^{o}=Q^{o T}>0 \\
A^{o} Q^{o}+Q^{o} A^{o T}+B^{o} Y^{o} C^{o}+C^{o T} Y^{o T} B^{o T}<0 . \\
C^{o} Q^{o}=H^{o} C^{o}
\end{gathered}
$$


When the above conditions hold, the common control law gain matrix is given by the equation

$$
K^{o}=Y^{o}\left(H^{o}\right)^{-1}
$$

Proof: Defining the Lyapunov function as follows

$$
v\left(q^{o}(t)\right)=q^{o T}(t) P^{o} q^{o}(t)>0
$$

where $P^{o}=P^{o T}>0$, then

$$
\dot{v}\left(\boldsymbol{q}^{\circ}(t)\right)=\dot{\boldsymbol{q}}^{\circ T}(t) \boldsymbol{P}^{\circ} \boldsymbol{q}^{\circ}(t)+\boldsymbol{q}^{\circ T}(t) \boldsymbol{P}^{\circ} \dot{\boldsymbol{q}}^{\circ}(t)<0
$$

Substituting (12) and (13) into (19) it yields

$$
\dot{v}\left(\boldsymbol{q}^{\circ}(t)\right)=\boldsymbol{q}^{\circ T}(t)\left(\boldsymbol{A}_{c}^{\circ T} \boldsymbol{P}^{\circ}+\boldsymbol{P}^{\circ} \boldsymbol{A}_{c}^{\circ}\right) \boldsymbol{q}^{\circ}(t)<0
$$

where

$$
A_{c}^{o}=A^{o}+B^{o} K^{o} C^{o}
$$

and (20) implies

$$
A_{c}^{o T} P^{o}+P^{o} A_{c}^{o}<0
$$

Since $P^{o}$ is positive definite, it also yields

$$
Q^{o} A_{c}^{o T}+A_{c}^{o} Q^{o}<0
$$

where $Q^{o}=\left(P^{o}\right)^{-1}$ and writing (23) in the open form, it is obtained

$$
Q^{o}\left(A^{o}+B^{o} K^{o} C^{o}\right)^{T}+\left(A^{o}+B^{o} K^{o} C^{o}\right) Q^{o}<0
$$

Analysing the matrix product in (24) it can be set

$$
B^{o} K^{o} C^{o} Q^{o}=B^{o} K^{o} H^{o}\left(H^{o}\right)^{-1} C^{o} Q^{o}
$$

where $H^{\circ}$ is a regular square matrix of appropriate dimension.

Defining the following equality

$$
\left(H^{o}\right)^{-1} C^{o}=C^{o}\left(Q^{o}\right)^{-1}
$$

and using the notation

$$
Y^{o}=K^{o} H^{o}
$$

then

$$
B^{o} K^{o} C^{o} Q^{o}=B^{o} Y^{o} C^{o}
$$

(24) implies (15) and (26) implies (16). This concludes the proof.

In practice, the case with $r=m$ (square plants) is often encountered, where it is generally associated with each output signal a reference signal, which is expected to influence as wanted this output. Such regime, reflecting nonzero set working points, is called the forced regime. 


\section{Definition 1}

The forced regime for (1), (2) with the bi-proper dynamic output controller (3),(4) is given by the control policy

$$
\begin{gathered}
\dot{\boldsymbol{p}}(t)=\boldsymbol{J} \boldsymbol{p}(t)+\boldsymbol{L} \boldsymbol{y}(t) \\
\boldsymbol{u}(t)=\boldsymbol{M p}(t)+\boldsymbol{N} \boldsymbol{y}(t)+\boldsymbol{W} \boldsymbol{w}(t)
\end{gathered}
$$

where $r=m, w(t) \in R^{m}$ is desired output signal vector, and $W \in R^{m \times m}$ is the signal gain matrix.

\section{Theorem 2}

If the system (1), (2) is stabilizable by the control policy (29), (30) and [13]

$$
\operatorname{rank}\left[\begin{array}{cc}
A & B \\
C & 0
\end{array}\right]=n+m
$$

then the matrix $W$ in (29), designed by using the static decoupling principle, takes the form

$$
W=-\left(C\left(A-B M J^{-1} L C+B N C\right)^{-1} B\right)^{-1}
$$

Proof: In a steady state which corresponds to $\dot{\boldsymbol{q}}(t)=\mathbf{0}, \dot{\boldsymbol{p}}(t)=\mathbf{0}$ the equality $y_{\mathrm{o}}=w_{\mathrm{o}}$ must hold. Denoting $q_{o} \in R^{n}, y_{o}, w_{o} \in R^{m}$ as the vectors of steady state values of $q(t), y(t), w(t)$, respectively, then (1) - (3) and (30) imply

$$
\begin{gathered}
0=A q_{o}+B u_{o} \\
0=J p_{o}+L C q_{o} \\
y_{o}=C q_{o} \\
u_{o}=M p_{o}+N y_{o}+W w_{o}
\end{gathered}
$$

Since now (34) - (36) implies

$$
u_{o}=\left(-M J^{-1} L C+N C\right) q_{o}+W w_{o}
$$

then, substituting (37) into (33), it yields

$$
\begin{aligned}
& 0=\left(A-B M J^{-1} L C+B N C\right) q_{o}+B W w_{o} \\
& q_{o}=-\left(A-B M J^{-1} L C+B N C\right)^{-1} B W w_{o}
\end{aligned}
$$

respectively, and with (35)

$$
y_{o}=-C\left(A-B M J^{-1} L C+B N C\right)^{-1} B W w_{o}
$$

Thus, considering $y_{o}=w_{o}$, then (40) implies (32). This concludes the proof.

The $W$ matrix is nothing else than the inverse of the closed-loop static gain matrix. This gain matrix can be obtained so by setting $s=0$ in the state-space expression of the transfer function matrix of the closed-loop system with respect to the forced input. Note, the static gain realized by the $W$ matrix is ideal in control only if the plant parameters, on which the value of $W$ depends, are known and do not vary with time. 
The forced regime is basically designed for constant references and is very closely related to shift of origin. If the command value $w(t)$ is changed "slowly enough," the above scheme can do a reasonable job of tracking, i.e., making $y(t)$ follow $w(t)$ [14].

In most cases the control using the non-proper dynamic output control is practically equivalent to the static output control, as the control law component defined by the output direct part $N y(t)$ is dominant. So the static output control is preferred, or the proper dynamic output control is fitted.

\section{STRICTLY PROPER DYNAMIC OUTPUT CONTROLLER}

Considering the strictly proper dynamic output controller, the following form of the closed-loop system description is obtained

$$
\begin{aligned}
& {\left[\begin{array}{c}
\dot{\boldsymbol{q}}(t) \\
\dot{\boldsymbol{p}}(t)
\end{array}\right]=\left[\begin{array}{cc}
\boldsymbol{A} & \boldsymbol{B M} \\
\boldsymbol{L} \boldsymbol{C} & \boldsymbol{J}
\end{array}\right]\left[\begin{array}{c}
\boldsymbol{q}(t) \\
\boldsymbol{p}(t)
\end{array}\right]} \\
& \boldsymbol{y}(t)=\left[\begin{array}{ll}
\mathbf{0} & \boldsymbol{I}_{m}
\end{array}\right]\left[\begin{array}{cc}
\mathbf{0} & \boldsymbol{I}_{n} \\
\boldsymbol{C} & \mathbf{0}
\end{array}\right]\left[\begin{array}{c}
\boldsymbol{q}(t) \\
\boldsymbol{p}(t)
\end{array}\right]
\end{aligned}
$$

Using the notations(10), (11) the closed-loop state-space equations takes the form

$$
\begin{gathered}
\dot{\boldsymbol{q}}^{\circ}(t)=\boldsymbol{A}_{c}^{\bullet} \boldsymbol{q}^{\circ}(t) \\
\boldsymbol{y}^{\circ}(t)=\boldsymbol{I}^{\circ} \boldsymbol{C}^{\circ} \boldsymbol{q}^{\circ}(t)
\end{gathered}
$$

where $I^{o}, C^{o}$ are given in (11) and

$$
A_{c}^{\bullet}=\left[\begin{array}{cc}
A & B M \\
L C & J
\end{array}\right]
$$

Note, only the square system $(r=m)$ is considered in the following.

\section{Theorem 3}

The strictly proper dynamic output controller (5), (6) to the system (1), (2) exists if there exist symmetric positive definite matrices $Q_{1}, S_{1} \in R^{n \times n}$ and matrices $L_{1} \hat{\mathrm{I}} R^{n^{\prime} m}, M_{1} \hat{\mathrm{I}} R^{r^{\prime} n}$ such that

$$
\begin{gathered}
Q_{1}=Q_{1}^{T}>0, \quad S_{1}=S_{1}^{T}>0, \quad\left[\begin{array}{cc}
S_{1} & I \\
I & Q_{1}
\end{array}\right]>0 \\
A Q_{1}+Q_{1} A^{T}+B M_{1}+M_{1}^{T} B^{T}<0 \\
S_{1} A+A^{T} S_{1}+L_{1} C+C^{T} L_{1}^{T}<0
\end{gathered}
$$

When the above conditions hold, the control law gain matrices are given as follows

$$
J=J_{1}\left(R_{1}-S_{1}^{-1}\right)^{-1}, \quad L=-S_{1}^{-1} L_{1}, \quad M=M_{1}\left(R_{1}-S_{1}^{-1}\right)^{-1}
$$

where 


$$
J_{1}=S_{1}^{-1}\left(A^{T}+\left(S_{1} A+L_{1} C\right) Q_{1}+S_{1} B M_{1}\right)
$$

Proof: Defining the Lyapunov function as in (18) then analogously can be obtained

$$
\begin{aligned}
& A_{c}^{\cdot T} P^{\circ}+P^{\circ} A_{c}^{\bullet}<0 \\
& Q^{\circ} A_{c}^{\cdot T}+A_{c}^{\circ} Q^{\circ}<0
\end{aligned}
$$

respectively, where $Q^{\circ}=\left(P^{\circ}\right)^{-1}$. Considering that

$$
Q^{\circ}=Q^{\circ}=\left[\begin{array}{ll}
Q_{1} & Q_{2} \\
Q_{2}^{T} & Q_{3}
\end{array}\right]>0
$$

hen the Schurov complement of (53) (with respect to $Q_{3}$ ) is

$$
S_{1}^{-1}=Q_{1}-Q_{2} Q_{3}^{-1} Q_{2}^{T}>0, \quad Q_{3}=Q_{3}^{T}>0
$$

and it can be set

$$
Q_{1}-S_{1}^{-1}=Q_{2} Q_{3}^{-1} Q_{2}^{T}>0
$$

Using $S_{1}^{-1}$, the following transform matrices can be defined

$$
T_{1}^{\circ}=\left[\begin{array}{cc}
S_{1} & S_{1} \\
I & 0
\end{array}\right], \quad T_{2}^{\circ}=\left[\begin{array}{cc}
I & 0 \\
0 & -Q_{2} Q_{3}^{-1}
\end{array}\right]
$$

and it yields

$$
\begin{gathered}
T_{1}^{\circ} T_{2}^{\circ} Q^{\circ} T_{2}^{\circ T} T_{1}^{\circ T}= \\
=\left[\begin{array}{cc}
S_{1} & S_{1} \\
I & 0
\end{array}\right]\left[\begin{array}{cc}
I & 0 \\
0 & -Q_{2} Q_{3}^{-1}
\end{array}\right]\left[\begin{array}{ll}
Q_{1} & Q_{2} \\
Q_{2}^{T} & Q_{3}
\end{array}\right]\left[\begin{array}{cc}
I & 0 \\
0 & -Q_{3}^{-1} Q_{2}^{T}
\end{array}\right]\left[\begin{array}{ll}
S_{1} & I \\
S_{1} & 0
\end{array}\right]= \\
=\left[\begin{array}{cc}
S_{1} & -S_{1} Q_{2} Q_{3}^{-1} \\
I & 0
\end{array}\right]\left[\begin{array}{ll}
Q_{1} & Q_{2} \\
Q_{2}^{T} & Q_{3}
\end{array}\right]\left[\begin{array}{cc}
S_{1} & I \\
-Q_{3}^{-1} Q_{2}^{T} S_{1} & 0
\end{array}\right]= \\
=\left[\begin{array}{cc}
S_{1} & -S_{1} Q_{2} Q_{3}^{-1} \\
I & 0
\end{array}\right]\left[\begin{array}{ll}
I & Q_{1} \\
0 & Q_{2}^{T}
\end{array}\right]=\left[\begin{array}{cc}
S_{1} & I \\
I & Q_{1}
\end{array}\right]>0
\end{gathered}
$$

where also (55) yields. Analogously,

$$
\begin{gathered}
T_{1}^{\circ} T_{2}^{\circ} A_{c}^{*} Q^{\circ} T_{2}^{\circ T} T_{1}^{\circ T}=\left[\begin{array}{cc}
S_{1} & -S_{1} Q_{2} Q_{3}^{-1} \\
I & 0
\end{array}\right]\left[\begin{array}{cc}
A & B M \\
L C & J
\end{array}\right]\left[\begin{array}{cc}
I & Q_{1} \\
0 & Q_{2}^{T}
\end{array}\right]= \\
=\left[\begin{array}{cc}
S_{1} A-S_{1} Q_{2} Q_{3}^{-1} L C & S_{1} B M-S_{1} Q_{2} Q_{3}^{-1} J \\
A & B M
\end{array}\right]\left[\begin{array}{cc}
I & Q_{1} \\
0 & Q_{2}^{T}
\end{array}\right]
\end{gathered}
$$

and denoting

$$
J_{1}=Q_{2} Q_{3}^{-1} J Q_{2}^{T}, \quad L_{1}=-S_{1} Q_{2} Q_{3}^{-1} L, \quad M_{1}=M Q_{2}^{T}
$$

(58) can be rewritten as follows 


$$
T_{1}^{\circ} T_{2}^{\circ} A^{\circ} Q^{\circ} T_{2}^{\circ T} T_{1}^{\circ} T=\left[\begin{array}{cc}
S_{1} A+L_{1} C & \left(S_{1} A+L_{1} C\right) Q_{1}+S_{1} B M_{1}-S_{1} J_{1} \\
A & A Q_{1}+B M_{1}
\end{array}\right]
$$

Finally, it yields

$$
\begin{aligned}
& T_{1}^{\circ} T_{2}^{\circ}\left(A_{c}^{\circ} Q^{\circ}+Q^{\circ} A_{c}^{\circ T}\right) T_{2}^{\circ T} T_{1}^{\circ T}= \\
& =\left[\begin{array}{cc}
S_{1} A+A^{T} S_{1}+L_{1} C+C^{T} L_{1}^{T} & U \\
U^{T} & A Q_{1}+Q_{1} A^{T}+B M_{1}+M_{1}^{T} B^{T}
\end{array}\right]<0
\end{aligned}
$$

where

$$
U=A^{T}+\left(S_{1} A+L_{1} C\right) Q_{1}+S_{1}\left(B M_{1}-J_{1}\right)
$$

Setting $U=0$, i.e.,

$$
S_{1} J_{1}=A^{T}+\left(S_{1} A+L_{1} C\right) Q_{1}+S_{1} B M_{1}
$$

then (61) imply (47), (48). It is evident that (47), (48) are conditioned by the inequalities (46) and (63) implies (50).

Writing as follows

$$
Q_{1}-S_{1}^{-1}=Q_{2} Q_{3}^{-1} Q_{2}^{T}=\left(Q_{1}-S_{1}^{-1}\right)\left(Q_{1}-S_{1}^{-1}\right)^{-1}\left(Q_{1}-S_{1}^{-1}\right)
$$

then with respect to (55) it can be set

$$
Q_{2} Q_{3}^{-1} Q_{2}^{T}=\left(Q_{1}-S_{1}^{-1}\right)\left(Q_{1}-S_{1}^{-1}\right)^{-1}\left(Q_{1}-S_{1}^{-1}\right)
$$

which leads to

$$
Q_{2}=Q_{2}^{T}=Q_{1}-S_{1}^{-1}, \quad Q_{3}=Q_{1}-S_{1}^{-1}, \quad Q_{2} Q_{3}^{-1}=I
$$

and, using(59), then (59) gives

$$
J_{1}=J\left(Q_{1}-S_{1}^{-1}\right), \quad L_{1}=-S_{1} L, \quad M_{1}=M\left(Q_{1}-S_{1}^{-1}\right)
$$

Thus, (67) implies (49). This concludes the proof.

\section{Definition 2}

The forced regime for (1), (2) with the strictly proper dynamic output controller (5), (6) is given by the control policy

$$
\begin{gathered}
\dot{\boldsymbol{p}}(t)=\boldsymbol{J} \boldsymbol{p}(t)+\boldsymbol{L} \boldsymbol{y}(t) \\
\boldsymbol{u}(t)=\boldsymbol{M} \boldsymbol{p}(t)+\boldsymbol{W} \boldsymbol{w}(t)
\end{gathered}
$$

where $w(t) \in R^{m}$ is desired output signal vector, and $W \in R^{m \times m}$ is the signal gain matrix.

\section{Theorem 4}

If the system (1), (2) is stabilizable by the control policy (68), (69) and satisfies (31) then the matrix $W$ in (69), designed by using the static decoupling principle, takes the form 


$$
W=-\left(C\left(A-B M J^{-1} L C\right)^{-1} B\right)^{-1}
$$

Proof: Setting $N=0$ in (32) then (70) is obtained. This concludes the proof.

\section{ILLUSTRATIVE EXAMPLE}

The features of the considered schemes and the effectiveness of the proposed design conditions are presented using the illustrative example.

The state space representation, describing the chemical reactor model [16], consists of the following matrices

$$
\boldsymbol{A}=\left[\begin{array}{rrrr}
1.380 & -2.080 & 6.715 & -5.676 \\
-0.581 & -4.290 & 0.000 & 0.675 \\
10.672 & 4.273 & -6.654 & 5.893 \\
0.482 & 4.273 & 1.343 & -2.104
\end{array}\right], \boldsymbol{B}=\left[\begin{array}{rr}
0.000 & 0.000 \\
5.679 & 0.000 \\
1.136 & -3.146 \\
1.136 & 0.000
\end{array}\right], \boldsymbol{C}^{T}=\left[\begin{array}{ll}
2 & 0 \\
0 & 0 \\
1 & 0 \\
0 & 1
\end{array}\right]
$$

and this system in the considered closed loop structures under the non-proper dynamic output control (3), (4) as well as under the proper dynamic output control (5), (6) was used in the presented simulations. Note, the pair $(A, B)$ is controllable and the pair $(A, C)$ is observable.

Within the above system parameters, solving (14)-(16) using the SeDuMi package for Matlab [17], the matrix parameters of the non-proper dynamic output controller were as follows

$$
\begin{gathered}
J=\operatorname{diag}\left[\begin{array}{llll}
-0.7195 & -0.7195 & -0.7195 & -0.7195
\end{array}\right], \quad N=\left[\begin{array}{lll}
1.2788 & -3.8548 \\
2.8385 & -5.9951
\end{array}\right] \\
M=10^{-10}\left[\begin{array}{llll}
-0.1464 & -0.0096 & -0.0244 & -0.0446 \\
-0.1889 & -0.1022 & -0.0432 & -0.0684
\end{array}\right], \quad L=10^{-10}\left[\begin{array}{lll}
-0.3131 & 0.5737 \\
-0.0831 & 0.0799 \\
-0.2439 & 0.6788 \\
-0.2996 & 0.8917
\end{array}\right]
\end{gathered}
$$

and the resulting global closed-loop system eigenvalues spectrum was

$$
\rho\left(A_{c}^{\circ}\right)=\{-0.0387,-0.0461,-0.0752 \pm 0.0770 \mathrm{i},-0.0072,-0.0072,-0.0072,-0.0072\}
$$

It is evident that in this case the static output control part, determined by the matrix $N$, is dominant.

Applying the same toolbox to solve LMIs (46)-(48) the obtained set of the proper dynamic controller matrix parameters was as follows

$$
J=\left[\begin{array}{rrrr}
-2.0338 & 0.2364 & 11.4248 & -6.4693 \\
-0.2450 & -0.0236 & -1.7606 & -9.5833 \\
-7.0794 & 7.1070 & -7.4777 & -2.5268 \\
1.1450 & 7.2147 & 4.2238 & -5.9784
\end{array}\right]
$$




$$
M=\left[\begin{array}{rrrr}
0.2797 & 1.0404 & -0.3355 & -1.5332 \\
2.9243 & -0.3763 & -2.6195 & 2.2187
\end{array}\right], \quad L=\left[\begin{array}{rr}
1.2432 & -3.8002 \\
0.6469 & 2.6497 \\
0.4235 & 4.7377 \\
-0.7341 & -1.3839
\end{array}\right]
$$

The both dynamic controller design methods, previously described, were applied to the simulation benchmark. The conditions in simulations were specified for system in the forced regimes, where

$$
\boldsymbol{p}^{T}(0)=\mathbf{0}, \quad \boldsymbol{q}^{T}(0)=\left[\begin{array}{llll}
0.1 & 0.1 & 0.0 & 0.0
\end{array}\right], \quad \boldsymbol{w}^{T}(t)=\left[\begin{array}{ll}
2 & 1
\end{array}\right]
$$

and the signal gain matrices $W_{d n}, W_{d p}$ were computed using (32), (70), respectively, as follows

$$
W_{d n}=\left[\begin{array}{ll}
-1.2128 & 3.8408 \\
-2.3680 & 5.9708
\end{array}\right], \quad W_{d p}=\left[\begin{array}{ll}
-0.0518 & 0.3335 \\
-0.3510 & 0.3335
\end{array}\right]
$$

Since the same desired output variables have been utilized to assess the each controller ability response and to demonstrate performance with respect to asymptotic properties, the results of the both proposed design method can be immediately compared.
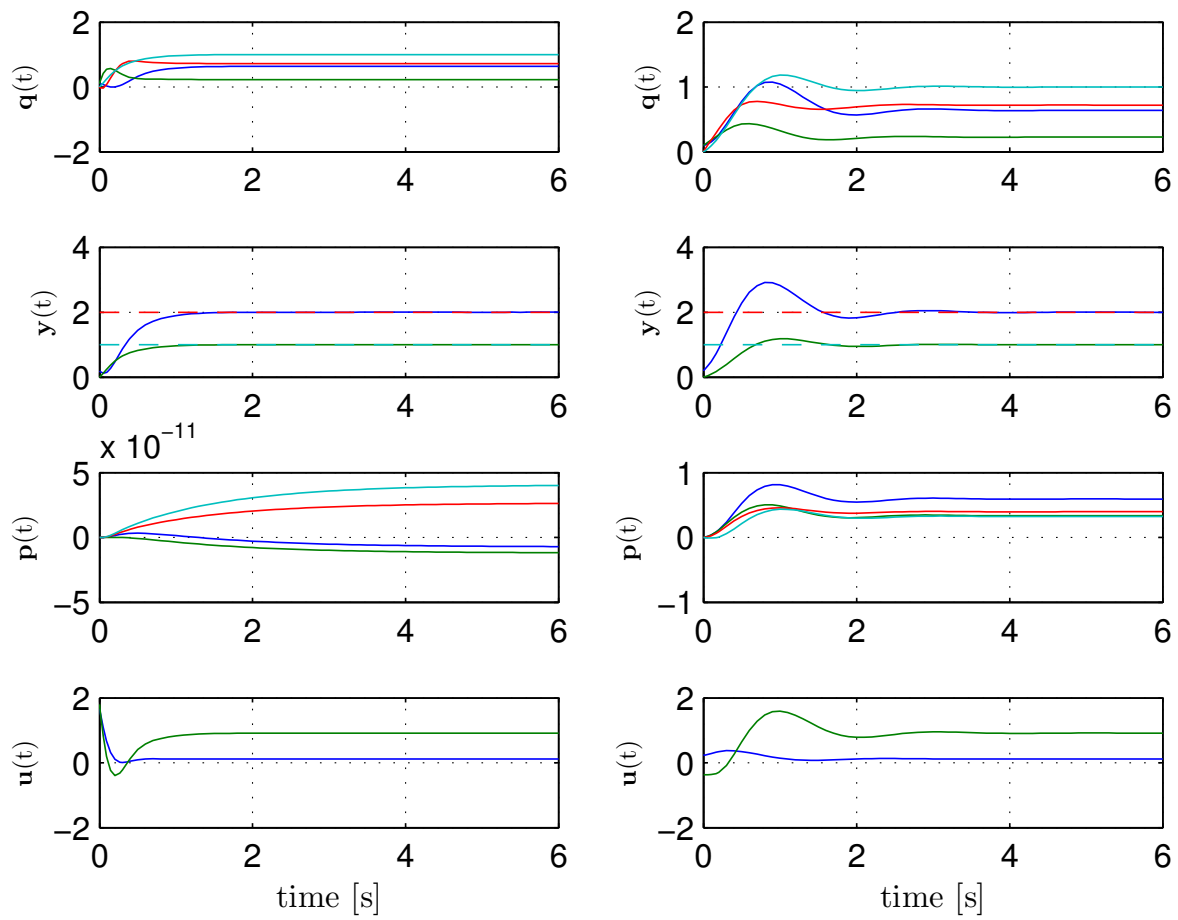

Figure 1: system variable responses using the dynamic output controllers

The left part of Fig. 1 shows the closed-loop system response with the dynamic output controller (3), (4) and the control policy (29), (30), which matrix parameter was obtained solving (14)-(16), (32). Using the dynamic output controller (5), (6) with the gain matrix parameters satisfying the conditions (46)-(48), (70), the right part of Fig. 1 shows the system response of the closed-loop system for the same system initial conditions and the control policy (68), (69). It is obvious from these figures that both controllers which parameters were obtained using the solutions of the LMI 
problems specified by Theorem 1 and Theorem 3 can successfully provide for the closed-loop system steady-state properties and asymptotic dynamics.

\section{CONCLUDING REMARKS}

New approach for output dynamic feedback control design is presented in this paper. By the proposed procedure the control problem is parameterized in such LMIs set with one additional LME which admit more freedom in guaranteeing the output feedback control performance for a bi-proper dynamic controller and by LMIs set only for a strictly proper dynamic output controller. Sufficient conditions of the controller existence manipulating the stability of the closed-loop systems imply the control structure, which stabilize the system in the sense of Lyapunov and the controller design tasks is a solvable numerical problem. An additional benefit of the method is that controller uses minimum feedback information with respect to desired system output and the approach is enough flexible to allow the inclusion of additional design condition bounds.

\section{ACKNOWLEDGMENTS}

The work presented in the paper was supported by VEGA, the Grant Agency of the Ministry of Education and the Academy of Science of Slovak Republic, under Grant No. 1/0348/14. This support is very gratefully acknowledged.

\section{REFERENCES}

[1] Chadli, M., Maquin, D. \& Ragot, J. (2002) "Static output feedback for Takagi-Sugeno systems: An LMI approach", In Proceedings of the 10th Mediterranean Conference on Control and Automation MED2002, Lisbon, Portugal.

[2] Syrmos, V.L., Abdallah, C. \& Dorato, P. (1994) "Static output feedback: A survey," In Proceedings of the 33rd IEEE Conference on Decision and Control, Lake Buena Vista, FL, USA, pp. 837-842.

[3] Syrmos, V.L., Abdallah, C., Dorato, P. \& Grigoriadis, K. (1997) "Static output feedback: A survey", Automatica, Vol. 33, No. 2, pp. 125-137.

[4] Krokavec, D. \& Filasova, A. (2013) "Design of fault residual functions for systems stabilized by static output feedback", In Proceedings of the 2nd International Conference on Control and Fault-Tolerant Systems SysTol'13, Nice, France, pp. 596-600.

[5] Astolfi, A. \& Colaneri, P. (2004) "The static output feedback stabilization problem as a concaveconvex programming problem", In Proceedings of the 2004 American Control Conference, Boston, MA, USA, pp. 2139-2141.

[6] Lewis, F.L. \& Syrmos, V.L. (1995) Optimal Control, New York, NY, USA, John Wiley \& Sons.

[7] Crusius, C.A.R. \& Trofino, A. (1999) "Sufficient LMI conditions for output feedback control problems", IEEE Transactions on Automatic Control, Vol. 44, No. 5, pp. 1053-1057.

[8] El Ghaoui, L. \& Balakrishnan, V. (1994) "Synthesis of fixed-structure controllers via numerical optimization", In Proceedings of the 33rd Conference on Decision and Control, Lake Buena Vista, FL, USA, pp. 2678-2683.

[9] Oliveira de, M.C., Camino, J.E. \& Skelton, R.E. (2000) "A convexifying algorithm for the design of structured linear controllers", In Proceedings of the 39th IEEE Conference on Decision and Control, Sydney, Australia, pp. 2781-2786.

[10] Scherer, C., Gahinet, P. \& Chilali, M. (1997) "Multiobjective output-feedback control via LMI optimization", IEEE Transactions on Automatic Control, Vol. 42, No. 7, pp. 896-911.

[11] Jungers, M., Castelan, E.B., Moraes, V.M. \& Moreno, U.F. (2013) "A dynamic output feedback controller for NCS based on delay estimates", Automatica, Vol. 49, No. 3, pp. 788-792.

[12] Krokavec, D. \& Filasova, A. (2007) Dynamic System Diagnosis. Kosice, Slovakia, Elfa. (in Slovak)

[13] Wang, Q.G. (2003) Decoupling Control. Berlin, Germany, Springer-Verlag.

[14] Kailath, T., (1980) Linear Systems, Englewood Cliffs, NJ, USA, Prentice-Hall. 
[15] Doyle, J.C., Glover, K., Khargonekar, P.P. \& Francis, B.A. (1989) "State-space solutions to standard H2 and Hœ control problems", IEEE Transactions on Automatic Control, vol. 34, no. 8, pp. 831-847, 1989.

[16] Kautsky, J., Nichols, N.K. \& Van Dooren, P. (1985) "Robust pole assignment in linear state feedback", International Journal of Control, Vol. 41, No.5, pp. 1129-1155.

[17] Peaucelle, D., Henrion, D., Labit, Y. \& Taitz, K. (2002) User's Guide for SeDuMi Interface 1.04, Toulouse, France, LAAS-CNRS.

\begin{abstract}
AUTHORS
Anna Filasova graduated in technical cybernetics and received M.Sc. degree in 1975, and Ph.D. degree in 1993 both from the Faculty of Electrical Engineering and Informatics, Technical University of Kosice, Slovakia. In 1999 she was appointed Associated Professor from the Technical University in Kosice in technical cybernetics. She is with the Department of Cybernetics and Artificial Intelligence, Faculty of Electrical Engineering and Informatics, Technical University of Kosice, and she has been working with as an Assistant Professor from 1975 to 1999. Her main research interests are in robust and predictive control, decentralized control, large-scale system optimization, and control reconfiguration.

Dusan Krokavec received M.Sc. degree in automatic control in 1967 and Ph.D. degree in technical cybernetics in 1982 from the Faculty of Electrical Engineering, Slovak University of Technology in Bratislava, Slovakia. In 1984 he was promoted Associated Professor from the Technical University in Kosice, Slovakia, and in 1999 he was appointed Full Professor in automation and control. He is with the Department of Cybernetics and Artificial Intelligence, Faculty of Electrical Engineering and Informatics, Technical University of Kosice. In the long term, he specializes in stochastic processes in dynamic systems, digital control systems and digital signal processing, and in dynamic system fault diagnosis.
\end{abstract}

